\title{
Neuropathie Sensitivo-Motrice Héréditaire avec ou sans Agénésie du Corps Calleux: Étude Radiologique et Clinique de 64 Cas
}

\author{
Jean Mathieu, Fernand Bédard, Claude Prévost et Pierre Langevin
}

\begin{abstract}
RÉSUMÉ: Neuropathie sensitivo-motrice héréditaire avec ou sans agénésie du corps calleux: étude radiologique et clinique de 64 cas Un syndrome autosomal récessif, retrouvé au sein d'une population originaire de Charlevoix et du Saguenay (Québec, Canada) et caractérisé par une agénésie du corps calleux (ACC) associée à une neuropathie sensitivo-motrice axonale, un retard intellectuel et quelques traits dysmorphiques, a été décrit par Andermann et Andermann en 1971. Les résultats de la tomodensitométrie cérébrale de 64 patients porteurs de ce syndrome révèlent une agénésie complète du corps calleux chez 37 patients $(57,8 \%)$, une agénésie partielle chez 6 patients $(9,4 \%)$ et la présence du corps calleux chez 21 patients $(32,8 \%)$. La présence du corps calleux a été confirmée dans 3 cas par un examen en résonance magnétique. La tomodensitométrie cérébrale a permis également de mettre en évidence une fréquence élevée d'anomalies développementaies ou dégénératives, en particulier l'élargissement de la fente interhémisphérique antérieure et l'atrophie ou l'hypodéveloppement de la fosse postérieure, malgré l'absence d'anomalie calleuse. La présentation clinique et l'histoire naturelle de l'atteinte polynévritique, intellectuelle et comportementale sont identiques chez les individus avec ou sans ACC. Des individus atteints de neuropathie sensitivo-motrice avec ou sans ACC se retrouvent au sein d'une même famille et souvent au sein d'une même fratrie. Ces observations supportent l'hypothèse d'un syndrome génétique unique dont l'expression phénotypique constante est la neuropathie sensitivomotrice.
\end{abstract}

\begin{abstract}
In 1971, Andermann and Andermann described an autosomal recessive syndrome found within the Charlevoix and the Saguenay populations (Quebec, Canada) characterized by agenesis of the corpus callosum (ACC) associated with motor and sensory neuropathy, mental retardation and dysmorphic features. A study of CT in 64 patients demonstrated a total ACC in 37 cases $(57.8 \%)$, partial ACC in 6 cases $(9.4 \%)$ and the presence of the corpus callosum in 21 cases $(32.8 \%)$. The latter was confirmed by MRI in 3 cases. CT of patients without ACC revealed a high frequency of developmental or degenerative midline anomalies, particularly interhemispheric fissure enlargement and posterior fossa atrophy. The clinical presentation and the natural course of the neuropathy, the intellectual impairment and the behavioural manifestations are identical amongst individuals with or without ACC. Individuals with or without ACC are found within the same family and often within the same sibship. These observations support the hypothesis of a single genetic syndrome in which the constant manifestation is the motor and sensory neuropathy.
\end{abstract}

Can. J. Neurol. Sci. 1990:17:103-108

Un syndrome autosomal récessif caractérisé par une agénésie du corps calleux (ACC), une neuropathie sensitivo-motrice avec quadriparésie flasque progressive, un retard intellectuel et un certain nombre de traits dysmorphiques a été décrit par Eva Andermann et Frédérick Andermann en 1971', $1975^{2}$ et 19793. Ce syndrome est rencontré essentiellement au sein de la population originant des régions de Charlevoix et du Saguenay-Lac-StJean, dans la province de Québec (Canada).

Chez de jeunes patients porteurs de ce syndrome, Larbrisseau et coll ${ }^{4}$ ont confirmé, par études histologiques et neurophysiologiques, une atteinte du nerf périphérique qui s'apparente à la neuropathie sensitivo-motrice héréditaire (NSMH) de type II (P.J. Dyck ${ }^{5}$ ).

Les publications d'Andermann et coll ainsi que celle de Larbrisseau font état d'une absence quasi-constante du corps calleux d'un individu atteint à l'autre. Ces même auteurs ont cependant rapporté quelques observations d'atteinte neuropathique typique du syndrome en présence d'un corps calleux normal.

Nous présentons ici les résultats de l'évaluation radiologique, clinique et généalogique de 64 personnes atteintes de cette neuropathie sensitivo-motrice héréditaire (NSMH) et habitant dans

De la Clinique des maladies neuro-musculaires (JM) et Service de génétique humaine (CP), Hôpital de Chicoutimi, Chicoutimi; Service de radiologie (FB), Hôpital Enfant-Jésus, Québec; Service de neuropédiatrie (PL), Centre Cardinal Villeneuve, Québec

Reçu le 19 juin 1989. Accepté le 18 decembre 1989

Tirés à part: Dr. Jean Mathieu, Clinique des maladies neuro-musculaires, Hôpital de Chicoutimi, 305 Saint-Vallier, Chicoutimi, PQ, Canada G7H 5 H6 
la région du Saguenay-Lac-St-Jean. Cette étude veut surtout attirer l'attention sur le manque de constance de l'ACC dans ce syndrome. Elle veut également comparer les modes de présentation et l'évolution clinique des patients avec et sans ACC afin de vérifier la valeur pronostique de cette anomalie cérébrale.

\section{Méthodologie}

Quatre-vingt-dix (90) patients atteints de NSMH avec ou sans ACC sont actuellement suivis à la Clinique des maladies neuro-musculaires de l'Hôpital de Chicoutimi. N'ont été retenus pour la présente étude que les 64 malades chez qui une tomodensitométrie cérébrale était disponible.

Cinquante-neuf (59) des tomodensitométries cérébrales ont été obtenues à l'Hôpital de Chicoutimi à l'aide d'un appareil Picker 1200, les cinq (5) autres à l'Hôpital Enfant-Jésus de Québec à l'aide d'un appareil Emi 5005 ou d'un appareil General Electric 9800 . Tous les examens ont été effectués en incidence axiale, et tous les films revus par un des auteurs (FB).

Les critères tomographiques suivants ont été retenus pour le diagnostic d'une agénésie totale du corps calleux:6-12

1. l'ascension, l'interposition et la dilatation du $3 \mathrm{e}$ ventricule;

2. l'élargissement de la fente interhémisphérique antérieure et sa juxtaposition à la portion antérieure du $3^{\mathrm{e}}$ ventricule;

3. l'écartement et le parallélisme des ventricules latéraux;

4. la déformation pointue, en corne, du rebord latéral des ventricules latéraux avec concavité interne (aspect en "chauve-souris");

5. l'agrandissement plus marqué du trigone et des cornes occipitales.

Pour l'agénésie partielle du corps calleux, nous avons retenu les critères radiologiques suivants: 6,8

1. I'agrandissement plus marqué du trigone et des cornes occipitales;

2. le parallélisme des ventricules latéraux;

3. la dilatation du $3^{\mathrm{e}}$ ventricule, surtout dans sa partie postérieure, avec parfois un aspect kystique du récessus suprapinéal.

Les données cliniques proviennent des dossiers médicaux de la Clinique des maladies neuro-musculaires de l'Hôpital de Chicoutimi. Les patients ont tous été examinés par un neurologue (JM), un orthopédiste et, pour les patients âgés de moins de 18 ans, par un pédiâtre.

Les données familiales et généalogiques ont été recueillies par le personnel de la Clinique, à l'aide d'un questionnaire d'histoire familiale complété avec la collaboration des membres

\begin{tabular}{lcccc}
\hline Tableau 1: Distribution Sexe/Age & & & \\
\hline & $\begin{array}{c}\text { NSMH } \\
\text { Avec } \\
\text { ACC }\end{array}$ & $\begin{array}{c}\text { NSMH } \\
\text { Sans } \\
\text { ACC }\end{array}$ & $\begin{array}{c}\text { NSMH } \\
\text { Avec } \\
\text { ACC } \\
\text { Partielle }\end{array}$ & Total \\
& $\mathrm{N}=37$ & $\mathrm{~N}=21$ & $\mathrm{~N}=6$ & $\mathrm{~N}=64$ \\
\hline $\begin{array}{l}\text { AGE (années) } \\
\quad \begin{array}{l}\text { Moyenne } \\
\text { (Ecart) }\end{array}\end{array}$ & 19.3 & 23.0 & 16.1 & 20.2 \\
$\begin{array}{l}\text { SEXE } \\
\text { H/F }\end{array}$ & $(2-34)$ & $(4-33)$ & $(2-31)$ & \\
$\quad$ Ratio & $24 / 13$ & $8 / 13$ & $1 / 5$ & $33 / 31$ \\
& 1,85 & 0,62 & & 1,06 \\
\hline
\end{tabular}

Tableau 2: Distribution Des Anomalies Radiologiques Cérébrales Autres que l'ACC

\begin{tabular}{|c|c|c|c|c|}
\hline & $\begin{array}{l}\text { NSMH } \\
\text { Avec } \\
\text { ACC } \\
\mathrm{N}=37\end{array}$ & $\begin{array}{l}\text { NSMH } \\
\text { Sans } \\
\text { ACC } \\
N=21\end{array}$ & $\begin{array}{c}\text { NSMH } \\
\text { Avec } \\
\text { ACC } \\
\text { Partielle } \\
\text { N }=6\end{array}$ & $\begin{array}{l}\text { Total } \\
N=64\end{array}$ \\
\hline $\begin{array}{l}\text { Agrandissement de la fente } \\
\text { inter-hémisphérique } \\
\text { antérieure }\end{array}$ & $*$ & 14 & & 14 \\
\hline Atrophie cérébelleuse & 3 & 7 & 1 & 11 \\
\hline $\begin{array}{l}\text { Hétérotopie de la substance } \\
\text { grise paraventriculaire }\end{array}$ & 2 & & & 2 \\
\hline $\begin{array}{l}\text { Augmentation du volume de } \\
\text { la grande citerne }\end{array}$ & 2 & 1 & & 3 \\
\hline Atrophie cérébrale diffuse & & 3 & & 3 \\
\hline $\begin{array}{l}\text { Augmentation des sillons } \\
\text { arachnoidiens frontaux }\end{array}$ & & & 1 & 1 \\
\hline $\begin{array}{l}\text { Anomalie du contour de la } \\
\text { corne frontale }\end{array}$ & & & 1 & 1 \\
\hline
\end{tabular}

*Anomalie qui fait partie des critères diagnostiques d'une ACC

des familles concernées. Certaines généalogies ont été remontées manuellement à l'aide d'un registre des mariages de Charlevoix. ${ }^{13}$ Enfin, d'autres liens d'apparentement entre personnes atteintes proviennent des dossiers généalogiques du Dr. Eva Andermann de l'Institut neurologique de Montréal.

\section{RÉSUltats}

\section{A) Tomodensitométrie cérébrale}

La tomodensitométrie cérébrale des 64 patients a permis d'identifier trente-sept (37) cas d'agénésie totale du corps calleux, six (6) cas d'agénésie partielle et vingt et un (21) cas ne démontrant aucun signe d'agénésie du corps calleux. Chez 3 des 21 patients qui ne présentent pas d'ACC à la tomodensitométrie cérébrale, l'intégrité du corps calleux a été confirmée par un examen en résonance magnétique.

Le Tableau 1 illustre la distribution sexe/âge des patients selon la présence ou l'absence d'une ACC. L'âge moyen est semblable dans les trois groupes; on note par ailleurs une prédominance nette des hommes (ratio $=1,85$ ) chez les individus avec $A C C$ et un résultat inverse (ratio $=0,62$ ) chez les patients sans $A C C\left(X^{2} 7.07 ; p=0.03\right)$.

De nombreuses anomalies cérébrales développementales ou dégénératives autres que l'ACC ont aussi été notées à la tomodensitométrie. Le Tableau 2 montre la distribution des anomalies radiologiques cérébrales les plus significatives. Chez les patients sans ACC, on observe une anomalie de la fosse postérieure dans $33 \%$ des cas $(7 / 21)$ dont une atrophie à prédominance vermienne inférieure dans trois cas. Les anomalies de la fosse postérieure sont moins nombreuses (3/37) chez les patients avec une ACC. L'élargissement de la fente interhémisphérique antérieure a été observé non seulement dans le majorité des cas d'ACC mais également chez 66\% (14/21) des patients sans ACC. Pour l'ensemble des 64 patients, $91 \%$ (58/64) présentent une ou plusieurs anomalies de la ligne médiane. 


\begin{tabular}{llccc}
\hline Tableau 3: Distribution des Anomalies Neurologiques et Squelettiques \\
\hline \hline
\end{tabular}

Tableau 4: Distribution des Anomalies Dysmorphiques, Intellectuelles et Comportementales

\begin{tabular}{|c|c|c|c|c|}
\hline & $\begin{array}{c}\text { NSMH } \\
\text { Avec } \\
\text { ACC }\end{array}$ & $\begin{array}{l}\text { NSMH } \\
\text { Sans } \\
\text { ACC }\end{array}$ & $\begin{array}{c}\text { NSMH } \\
\text { Avec } \\
\text { ACC } \\
\text { Partielle }\end{array}$ & Total \\
\hline & $\mathrm{N}=37$ & $\mathrm{~N}=21$ & $N=6$ & $\mathrm{~N}=64$ \\
\hline \multicolumn{5}{|l|}{$\begin{array}{l}\text { Principaux traits } \\
\text { dysmorphiques }\end{array}$} \\
\hline Palais ogival & 13 & 9 & 3 & 25 \\
\hline Brachycéphalie & 7 & 3 & 0 & 10 \\
\hline $\begin{array}{l}\text { "Overriding" du le orteil } \\
\text { Implantation basse du }\end{array}$ & 6 & 3 & 1 & 10 \\
\hline $\begin{array}{l}\text { pouce } \\
\text { Syndactylie partielle } 2^{\mathrm{c}-3 \mathrm{e}}\end{array}$ & 5 & 5 & 0 & 10 \\
\hline orteils & 2 & 2 & 1 & 5 \\
\hline \multicolumn{5}{|l|}{ Capacités intellectuelles } \\
\hline Intelligence normale & 3 & 1 & 0 & 4 \\
\hline Retard mental léger & 12 & 11 & 3 & 26 \\
\hline Retard mental modéré & 13 & 8 & 0 & 21 \\
\hline Retard mental profond & 1 & 0 & 1 & 2 \\
\hline Pas d'évaluation & 8 & 1 & 2 & 11 \\
\hline \multicolumn{5}{|l|}{ Troubles comportementaux } \\
\hline $\begin{array}{l}\text { Episode(s) psychotique(s) } \\
\text { après l'âge de } 15 \text { ans }\end{array}$ & $8 / 26$ & $8 / 18$ & $2 / 3$ & $18 / 47$ \\
\hline
\end{tabular}

Parmi les 64 sujets de la présente étude, onze (11) présentent une atrophie cérébelleuse; dix (10) des ces 11 sujets sont âgés de plus de 20 ans (âge moyen $=27$ ans). Cette observation suggère une étiologie dégénérative plutôt que développementale.

\section{B) Données cliniques}

Le mode de présentation et l'évolution clinique des 64 sujets atteints de ce syndrome sont similaires aux observations faites par Andermann ${ }^{2}$ et Larbrisseau. ${ }^{4}$ Tous les patients présentent une hypotonie aréflexique durant l'enfance avec retard des

\begin{tabular}{|c|c|c|}
\hline & $\begin{array}{l}\text { NSMH } \\
\text { Avec } \\
\text { ACC }\end{array}$ & $\begin{array}{l}\text { NSMH } \\
\text { Sans } \\
\text { ACC }\end{array}$ \\
\hline $\begin{array}{l}\text { Apparition de la marche } \\
\text { Age moyen (année) } \\
\text { (Ecart) }\end{array}$ & $\begin{array}{c}N=29 \\
3.5 \\
(1,5-6,5)\end{array}$ & $\begin{array}{l}N=18 \\
4,4 \\
(1,5-9)\end{array}$ \\
\hline $\begin{array}{l}\text { Arrêt de la marche } \\
\text { Age moyen (année) } \\
\text { (Ecart) }\end{array}$ & $\begin{array}{l}N=22 \\
12,6 \\
(3-27)\end{array}$ & $\begin{array}{l}N=13 \\
15.8 \\
(6-28)\end{array}$ \\
\hline $\begin{array}{l}\text { Apparition de la scoliose } \\
\text { Age moyen (année) } \\
\text { (Ecart) }\end{array}$ & $\begin{array}{l}N=14 \\
9,9 \\
(4-16)\end{array}$ & $\begin{array}{l}N=8 \\
11,3 \\
(1-25)\end{array}$ \\
\hline $\begin{array}{l}\text { Apparition des manifesta } \\
\text { psychotiques } \\
\text { Age moyen (année) } \\
\text { (Ecart) }\end{array}$ & $\begin{array}{l}N=8 \\
20,0 \\
(15-24)\end{array}$ & $\begin{array}{l}N=8 \\
22.6 \\
(17-30)\end{array}$ \\
\hline $\begin{array}{l}\text { Décès } \\
\text { Age moyen (année) } \\
\text { (Ecart) }\end{array}$ & $\begin{array}{l}N=5 \\
24,6 \\
(19-30)\end{array}$ & $\begin{array}{c}\mathrm{N}=1 \\
26,0\end{array}$ \\
\hline
\end{tabular}

acquisitions motrices. La marche apparaît vers l'âge de 3 à 5 ans, très souvent à l'aide d'orthèses aux membres inférieurs et d'un cadre de marche. On note ensuite une perte graduelle des habilités motrices avec amyotrophie de la musculature distale, contractures des extrémités et scoliose progressive. La majorité des patients sont confinés au fauteuil roulant vers l'âge de 12 à 15 ans. Le décès survient habituellement entre l'âge de 25 à 30 ans, rarement après l'âge de 40 ans.

La plupart des patients présentent un retard intellectuel de léger à modéré et un certain nombre de traits dysmorphiques dont une brachycéphalie, une asymétrie faciale, une ptose palpébrale, un hypertélorisme, un palais ogival, une implantation basse du pouce, une syndactylie partielle des $2^{c}$ et $3^{c}$ orteils et un superposition du le orteil sur le $2 \mathrm{e}$. Un comportement psychotique va se manifester chez $38.2 \%$ (18/47) des patients après l'âge de 15 ans. Ces épisodes psychotiques, de durée très variable, se caractérisent soit par un isolement autistique, soit par un délire paranoïde, soit par un état dépressif majeur, et s'accompagnent d'hallucinations visuelles ou auditives, d'une régression psycho-motrice importante avec incontinence urinaire ou fécale et d'une perte majeure d'autonomie.

Les Tableaux 3 et 4 illustrent la distribution des anomalies neurologiques, musculo-squellettiques, dysmorphiques, intellectuelles et comportementales des patients selon la présence ou l'absence du corps calleux.

La sévérité de l'atteinte polynévritique et de ses séquelles musculosquellettiques est identique dans les trois groupes de patients: il ne semble donc pas y avoir de relation entre cette sévérité et la présence ou l'absence du corps calleux. Les anomalies des paires crâniennes et la présence de crises convulsives sont distribuées également chez les patients avec et sans ACC. Il en est de même pour la présence des principaux traits dysmorphiques tels qu'observés par des cliniciens non-généticiens.

L'évaluation des capacités intellectuelles, effectuée selon la classification clinique de Taft, 14 montre une fréquence élevée (47/53) de retard mental de léger à modéré; il n'y a toutefois aucune association entre ce retard et la présence ou l'absence du 
corps calleux. Des études psychométriques comparatives entre les patients avec et sans $A C C n$ 'ont cependant pas été réalisées à ce jour. D'autre part, le présence ou l'absence du corps calleux n'influence ni la fréquence ni la sévérité des manifestations psychotiques.

L'étude comparative de l'évolution clinique des patients (Tableau 5) ne montre pas de relation significative entre la présence ou l'absence du corps calleux et l'âge d'apparition de la marche, l'âge d'arrêt de la marche, l'âge d'apparition de la scoliose, l'âge d'apparition des manifestations psychotiques ou l'âge au décès.

\section{C) Données généalogiques}

Quarante-et-un (4l) des 64 sujets de la présente étude sont apparentés (ancêtres communs situés entre la $1^{\mathrm{c}}$ et la $4^{\mathrm{e}}$ générations) à une autre personne atteinte de cette même neuropathie évolutive. L'analyse de la distribution intra-familiale des patients a été faite en fonction de la présence ou de l'absence du corps calleux.

Dix-huit (18) patients se retrouvent à l'intérieur de sept fratries; trois fratries regroupent à la fois des sujets atteints avec ACC et sans ACC. La Figure la illustre deux de ces fratries réunies au sein d'une même famille élargie. Lorsque le lien d'apparentement entre les sujets atteints est plus éloigné, on observe à nouveau des patients avec ou sans $\mathrm{ACC}$ au sein d'une même famille élargie. Cette observation se vérifie dans 5 des 9 généalogies qui ont été reconstituées.

Jusqu'à maintenant, aucun cas d'ACC sans neuropathie sensitivo-motrice n'a été identifié à l'intérieur de ces familles; il faut cependant noter que les personnes qui ne montrent aucun signe de neuropathie périphérique n'ont pas subi de tomodensitométrie cérébrale. A l'exclusion des patients porteurs du syndrome, on ne relève pas dans ces familles un taux anormalement élevé de déficience intellectuelle ou de troubles psychotiques.

\section{Discussion}

La tomodensitométrie cérébrale de soixante-quatre (64) cas saguenéens de NSMH a révélé que seulement $57,8 \%$ d'entre eux présentent une agénésie totale du corps calleux, alors que 32,8\% n'ont pas d'ACC et que 9,4\% ont une agénésie partielle. Larbrisseau ${ }^{4}$ avait déja noté, dans une étude de 15 sujets atteints de cette neuropathie, une agénésie partielle du corps calleux dans 3 cas $(20 \%)$ et un corps calleux normal chez 2 patients $(13,3 \%)$. Andermann et coll ${ }^{3}$ ont également rapporté au moins 5 cas typiques du syndrome neuro-musculaire avec présence du corps calleux. L'agénésie du corps calleux ne représente donc plus un critère diagnostique absolu pour cette affection héréditaire.

Dans ce syndrome saguenéen, dont la fréquence est égale pour les deux sexes, une ACC s'observe plus fréquemment chez les hommes. A l'exception des syndromes décrits par Aicardi14 et par Menkes, 15 un indice comparatif différent entre les deux sexes est rarement observé dans l'ACC. Pour l'instant, la signification de cette surreprésentation masculine avec ACC est incertaine.

Les anomalies cérébrales associées à l'agénésie du corps calleux, telles que décrites par Bird, ${ }^{6}$ Kendall ${ }^{8}$ et Sarwar, ${ }^{9}$ sont

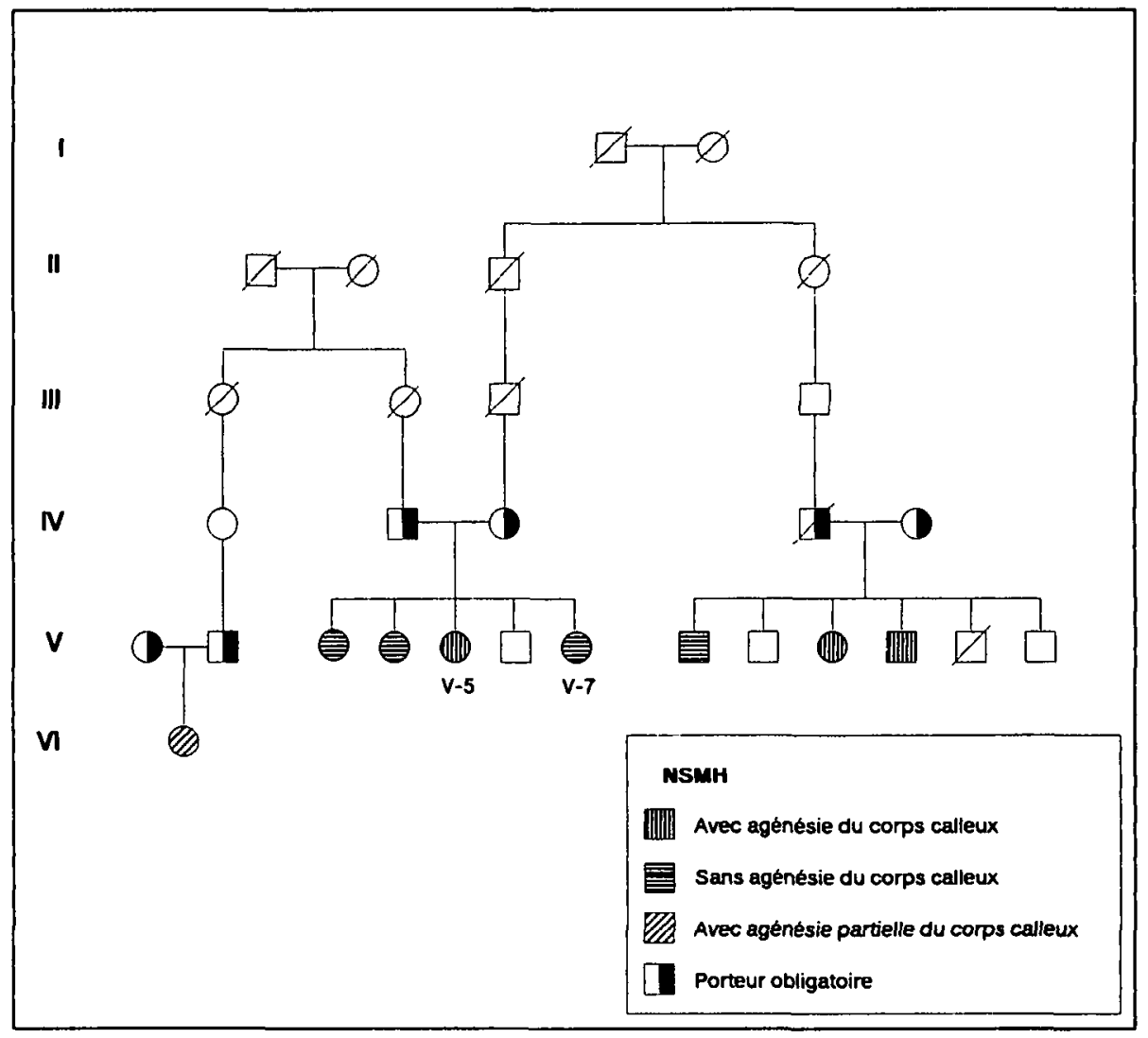

Figure la-Arbre généalogique de huit (8) individus atteints de NSMH avec ou sans ACC. 


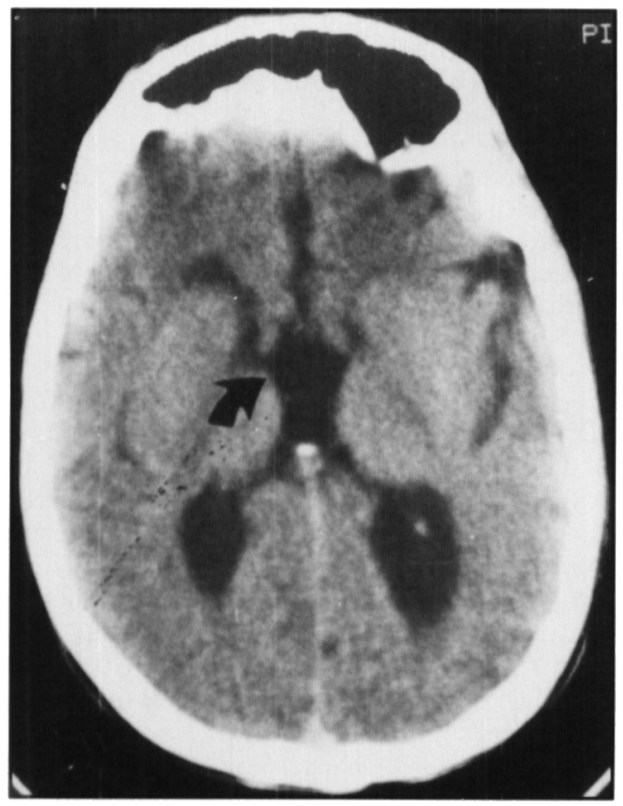

Figure $I b-I n d i v i d u$ V-5. Image classique d'une agénésie du corps calleux à la tomodensitométrie cérébrale. Remarquez le contact de la fente interhémisphérique avec le 3e ventricule dilaté.

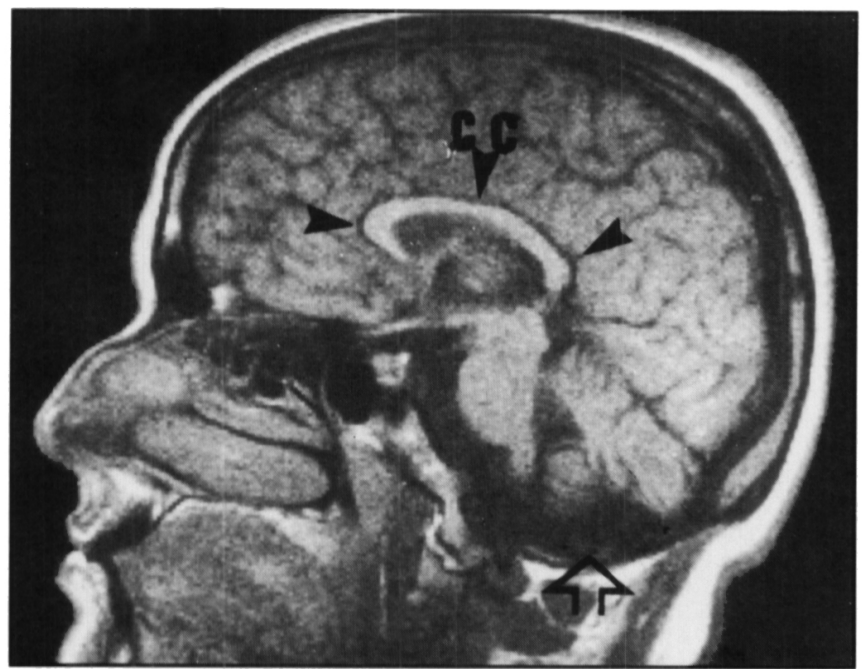

Figure Ic - Individu V-7. Corps calleux normal et atrophie du vermis inférieur à l'examen en résonance magnétique.

différentes des anomalies de la ligne médiane retrouvées chez nos sujets atteints de NSMH avec ACC. En effet, nous n'avons noter aucun cas de lipome du corps calleux, de malformation de Chiari type 2 ou de malformation de Dandy Walker. Par ailleurs, les sujets atteints d'une NSMH sans ACC présentent souvent d'autres anomalies de la ligne médiane dont une atrophie de la fosse postérieure (Figure lc) ou un élargissement de la fente inter-hémisphérique antérieure (Figure 2).

L'élargissement de la fente inter-hémisphérique antérieure et sa proximité avec le $3 \mathrm{e}$ ventricule sont habituellement associés aux autres anomalies radiologiques typiques de l'agénésie du corps calleux (Sarwar ${ }^{9}$ ). Cependant, la présence isolée d'un élargissement de la fente inter-hémisphérique antérieure sans contact de celle-ci avec le $3^{\mathrm{e}}$ ventricule pourrait, tout comme le mentionne Sarwar, ${ }^{9}$ représenter une forme mineure de "dysgénésie du corps calleux"; on ne peut cependant classifier ces patients dans le groupe des NSMH avec ACC en l'absence des autres critères radiologiques primordiaux. Nous croyons que cet élargissement de la fente interhémisphérique antérieure indique soit une anomalie de développement, soit une atrophie des lobes frontaux, et doit être considéré comme un type d'anomalie de la ligne médiane associé à la NSMH.

La troisième anomalie de la ligne médiane la plus fréquemment retrouvée dans ce syndrome serait l'atrophie ou l'hypodéveloppement cérébelleux, en particulier du vermis inférieur (Figure lc).

L'analyse de la distribution intra-familiale des patients en fonction de la présence ou de l'absence du corps calleux révèle une hétérogénéité importante, la moitié des familles combinant à la fois des cas de NSMH avec et sans ACC. De plus, des individus avec ou sans $A C C$ se retrouvent au sein de mêmes fratries. Ces observations supportent l'hypothèse d'un syndrome génétique unique dont l'expressivité phénotypique constante n'est pas l'ACC mais plutôt la neuropathie sensitivo-motrice. Cette neuropathie est associée à plusieurs anomalies cérébrales, notamment à diverses anomalies de la ligne médiane dont les plus fréquentes sont l'agénésie plus ou moins complète du corps calleux, l'élargissement de la fente inter-hémisphérique antérieure et l'atrophie cérébelleuse.

Certaines des anomalies cérébrales associées à ce syndrome, dont l'ACC, sont d'origine développementale, d'autres, dont l'atrophie cérébelleuse, semblent d'origine dégénerative. Plusieurs pathologies métaboliques ont été décrites en association avec des malformation ou anomalies de structure cérébrale, dont l'hyperglycinémie non-cétonique, le syndrome de Zellweger, le déficit en pyruvate dehydrogénase et l'adréno-

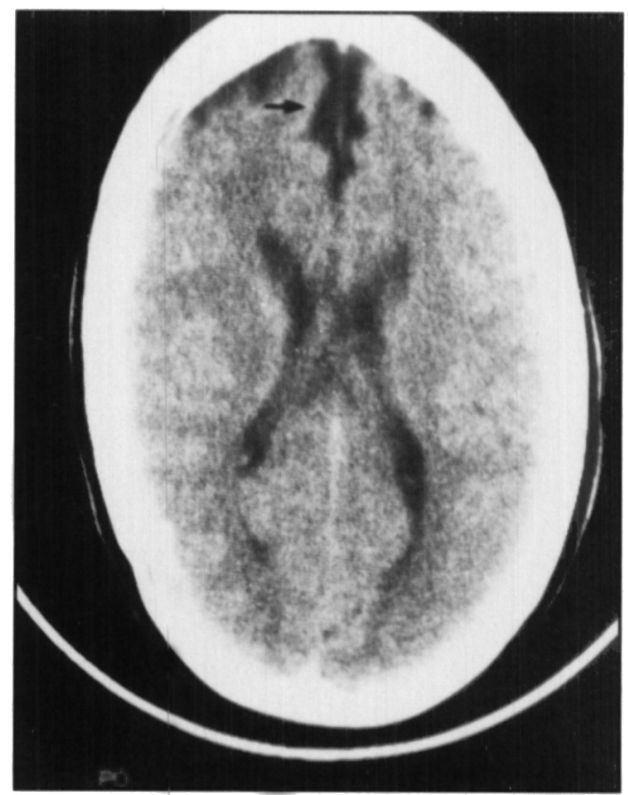

Figure 2 - Élargissement de la fente inter-hémisphérique antérieure à la tomodensitométrie cérébrale chez une patiente atteinte d'une NSMH sans ACC. 
leukodystrophie néonatale.17,18 A ce jour, aucun déficit métabolique n'a été identifié dans ce syndrome saguenéen. Cependant, la présence d'une neuropathie évolutive, d'anomalies cérébrales dégénératives surajoutées aux malformations cérébrales ainsi que la présence d'une modification progressive du comportement avec manifestations psychotiques orientent nettement vers une atteinte de type métabolique.

La sévérité de l'atteinte polynévritique, intellectuelle, dysmorphique et comportementale n'est pas liée à la présence ou à l'absence du corps calleux. Cette anomalie cérébrale n'a donc pas d'utilité pronostique dans ce syndrome. Par ailleurs, les aspects cliniques n'ont pas été étudiés en fonction des autres anomalies radiologiques de la ligne médiane décrites dans la présente étude.

Ce syndrome récessif doit donc être soupçonné chez un enfant porteur d'une atteinte polynévritique, originaire de Charlevoix ou du Saguenay, malgré la présence d'un corps calleux démontrée par tomodensitométrie ou en résonance magnétique. Dans ce contexte clinique, l'histoire familiale exhaustive, la recherche de traits dysmorphiques et l'identification d'anomalies radiologiques, entre autres de la ligne médiane, permettront un diagnostic précoce de ce syndrome et un conseil génétique approprié. Par ailleurs, l'absence d'anomalie calleuse observée chez plusieurs malades réduit sensiblement la valeur diagnostique d'un dépistage anténatal par échographie.

\section{REMERCIEMENTS}

Les auteurs remercient le Dr. Marc DeBraekeleer pour les études statistiques et le Dr. Xavier Prat pour les examens effectués à l'aide de la résonance magnétique. Nous remercions également le Dr. Nicolas Daoud, M. Guy Gagnon et Mme Claire Potvin de l'Hôpital de Chicoutimi pour leur patiente collaboration à la réalisation des tomodensitométries cérébrales.

\section{RÉFÉRENCES}

1. Andermann E, Andermann F, Melançon D. Three familial midline malformation syndromes of the central nervous system. Proceedings of the Fourth International Congress of Human Genetics, Ed. J. de Grouchy, Amsterdam. Excerpta Medica International Congress Series 1971; 233: 15.

2. Andermann $E$, Andermann $F$, Joubert $M$, et al. Three familial midline malformation syndromes of the central nervous system: age- nesis of the corpus callosum and anterior horn-cell disease; agenesis of the cerebellar vermis; and atrophy of the cerebellar vermis. Birth Defects: original article series 1975; XI: 2.

3. Andermann E, Andermann F, Bergeron D, et al. Familial agenesis of the corpus callosum with sensorimotor neuropathy: genetic and epidemiological studies over 170 patients. Can J Neurol Sci 1979; 6: 400.

4. Larbrisseau A, Vanasse M, Brochu P, et al. The Andermann Syndrome: agenesis of the corpus callosum associated with mental retardation and progressive sensorimotor neuropathy. Can $\mathrm{J}$ Neurol Sci 1984; 11: 257-261.

5. Dyck PJ. Inherited neuronal degeneration and atrophy affecting peripheral motor, sensory and autonomic neurons. In: Dyck PJ, Thomas PK, Lambert EH, Bunge R, eds. Peripheral Neuropathy, Vol. II. Philadelphia: W.B. Saunders Co. 1984; 1600-1655.

6. Bird SE, Harwood-Nash AC, Fitz CR. Absence of the corpus callosum: computed tomographic evaluation in infants and children. J Can Assoc Radiol 1978; 29: 108-112.

7. Besigye E. Diagnosis of agenesis of the corpus callosum by computed assisted tomography: report of two cases presenting with epileptic seizures. Comput Tomogr 1979; 3: 155-158.

8. Kendall BE. Dysgenesis of the corpus callosum. Neuroradiology 1983; 25: 239-256.

9. Sarwar M, Virapongse C, Bhimani S, et al. Interhemispheric fissure sign of dysgenesis of the corpus callosum. J Comput Assist Tomogr 1984; 8: 637-644.

10. Davidson HD, Abraham R, Steinor RE. Agenesis of the corpus callosum: magnetic resonance imaging. Radiology 1985; 155: 371373.

11. McLeod NA, Powell Williams J, Machen B, et al. Normal and abnormal morphology of the corpus callosum. Neurology 1987; 37: 1240-1242.

12. Harwood-Nash DC, Fitz CR. Neuroradiology in infants and children. St-Louis: CV Mosby 1976.

13. Talbot EG. Généalogies pour les comtés de Charlevoix et de Saguenay (6 tomes). Québec: Chateau-Richer 1978-79.

14. Taft LT. Mental retardation: an overview. Pediatr Ann 1973; 2: 1024.

15. Aicardi J, Chevrie JJ, Rousselie F. Le syndrome spasmes en flexion, agénésie calleuse, anomalies chorio-rétiniennes. Arch Franc Pédiat 1969; 26: 1103-1120.

16. Menkes JH, Phillipart M, Clark DB. Hereditary partial agenesis of the corpus callosum. Arch Neurol 1964; 11: 198-208.

17. Dobyns WB. Agenesis of the corpus callosum and gyral malformations are frequent manifestations of nonketotic hyperglycinemia. Neurology 1989; 39: 817-820.

18. Kolodny EH. Agenesis of the corpus callosum: a marker for inherited metabolic disease? Neurology 1989; 39: 847-848. 